Beate Kierey

\title{
Wickel und Auflagen als äussere Anwendungen in der ambulanten Pflege: Weisskohlauflage
}

\section{Traditionelle äussere Anwendungen}

$\mathrm{Zu}$ den häufig durchgeführten äusseren Anwendungen gehört beispielsweise ein Senfmehl-Fussbad oder ein Öldispersionsbad nach Werner Junge, ein Dampfbad mit Kamillenblüten zur Linderung bei Sinusitis, Husten oder einer Bronchitis, Wickel wie der Brust- oder Halswickel, kleine Wickel um Gelenke sowie rhythmische Einreibungen. Auflagen, die mit einer Salbe wie Aurum/ Lavandula comp. Creme von Weleda (Arlesheim, Schweiz) versehen sind, erweisen sich wirkungsvoll bei vegetativen Herz-Kreislauf-Beschwerden, Quarkauflagen bei Brustdrüsenentzündungen, und Wirsing- oder Weisskohlauflagen entfalten ihre Wirkungskräfte unter anderem bei kleineren Wunden und Entzündungen.

Mithilfe äusserer Anwendungen gelangen wirksame, heilende Substanzen aus Essenzen, Tees, Ölen oder Salben in den Körper und aktivieren, beruhigen oder entspannen die betreffenden Stellen.

\section{Wickel und Auflagen in der ambulanten Pflege}

Viele Wickel und Auflagen sind aus der Grosselterngeneration bekannt. Es ist interessant $\mathrm{zu}$ beobachten, dass gerade diese «alten» Naturheilmethoden in unserer modernen Gesellschaft ein Comeback feiern. Auch in der ambulanten Pflege, wie der Lukaspflege Freiburg e.V., die um die anthroposophische Pflege erweitert ist, stellen Bäder bzw. Teilbäder wie das Fussbad, rhythmische Einreibungen nach Wegman/Hauschka sowie Wickel und Auflagen eine wertvolle und wirksame Hilfe dar.

Hermann Glaser macht in seinem «Handbuch Gesundheitspflege» [1] deutlich, dass es einen gravierenden Unterschied zwischen Wickeln und Auflagen gibt. "Obwohl der Begriff Wickel sehr umfassend, auch als Überbegriff für alle äußeren Anwendungen gebraucht wird, bezeichnet er im Grunde nur die zirkuläre Einhüllung (also wirklich rundherum) eines Körperteils mit einer bestimmten Substanz» [1]. Eine Auflage, wie in diesem Artikel als Praxisbeispiel gezeigt, wird lediglich für eine «örtliche Substanzverabreichung» verwendet. Aussentücher bzw. Binden können aber in jedem Falle rundherum angelegt werden.

\section{Die Haut - Pforte für äussere Anwendungen}

Die Haut ist die Pforte für alle äusseren Anwendungen. Sie nimmt die Berührung bei der Einreibung sowie die angewendeten Substanzen auf und leitet sie weiter. Gerade bei älteren Menschen reagiert sie besonders auf Wärme- und Kälteimpulse; die Reak- tion reicht dabei von stumpf bis sehr empfindlich. Fällt eine nur schwache Reaktion auf die Anwendung aus, ist eine regelmässige Wiederholung günstig, damit die Haut allmählich für eine feinere Wahrnehmung sensibilisiert wird und Selbstheilungsprozesse angeregt werden. Allgemein lässt sich feststellen, dass eine Anwendung besonders wirkungsvoll ist, wenn sie in einem gewissen Rhythmus erfolgt, dadurch werden die Stoffwechselprozesse unterstützt, ein entspanntes Aus- und Einatmen wird spürbar und damit auf seelischer, körperlicher und geistiger Ebene ein Linderungsprozess eingeleitet.

Eine nicht unwichtige Rolle spielt dabei auch die «direkte Zuwendung des Pflegenden zu dem zu begleitenden Menschen» [2]. Denn durch die Anwendung, die Berührung, die direkte Hinwendung zum Menschen entsteht immer wieder ein besonderer Moment, der letztendlich ebenfalls die Sinne berührt. Gerade betagte Menschen, die oft in einer isolierten Situation und nur in ihrer gewohnten Umgebung/Wohnung leben und wenig Kontakt zur Aussenwelt haben, erfahren einerseits durch die Hinwendung, andererseits aber auch durch den Duft der Öle und Salben neue Reize, Anregungen und eine innere Bewegung.

So kann ein Fussbad mit Dr. Hauschka Citronenbad erfrischen, ein Bad mit Fichtennadel ausgleichen und ein Rosmarin-Bad beleben und wärmen.

\section{KARGER}

Fax +497614520714 (c) 2016 S. Karger GmbH, Freiburg

Accessible online at:

www.karger.com/szg
Beate Kierey

Lassbergstrasse 24, 79117 Freiburg, Deutschland b.kierey@t-online.de 


\section{Weisskohl- oder \\ Wirsingauflage in der äusseren Anwendung}

Kohl als Auflage zeigt sich besonders wirkungsvoll bei entzündlichen oder geschwollenen Gelenken im Fall von Arthritis und Rheuma, schlecht heilenden, nekrotischen und belegten Wunden, Venenentzündung und Dekubitus [3]. «Schon im Altertum galt die Kohlpflanze als Universalheilmittel», bemerkt Herrmann Muck [4], "... weil die Römer das gekochte Kohlblatt als inneres und äußeres Reinigungsmittel, für Umschläge, zur Wundbehandlung der Legionäre und gegen die Pest anwendeten». Gemäss Hermann Glaser fördert der Weisskohl «die Eiter- und Flüssigkeitsbildung und -ableitung nach außen über die Haut. Er wirkt kühlend, und beruhigend auf allgemein entzündliche Prozesse, verhärtete Wunden werden aufgeweicht, Verkrustungen können sich lösen und Wundsekret abfließen» [5].

Technik der äusseren Anwendung

Weisskohl- oder Wirsingblätter werden möglichst «aus biologischdynamischem Anbau verwendet, da der Kohl aus konventionellem Anbau unter Umständen zu sehr belastet» [6] ist.

Weiter benötigt wird ein Messer, ein Brett, Frischhaltefolie oder eine Gefrierbeuteltüte, ein Wellholz oder eine Glasflasche (denn sie saugt den Saft des Kohls nicht auf), eine elastische Binde und eventuell ein Tuch zum Umhüllen.

\section{Vorgehen}

Die äusseren Blätter des Kohls werden entfernt und einige davon entsprechend der zu behandelnden Fläche für die Anwendung verwendet. Bei einem Dekubitus kann unter Umständen schon die Grösse eines CentStücks ausreichen. Der Kohl wird unter lauwarmem Wasser gewaschen und im Anschluss getrocknet. Die harte Blattachse wird herausgeschnitten (harte Blattachsen verursachen während der Auflage oft Schmerzen) (Abb. 1). Nun wird das Kohlblatt gewalkt oder geklopft. Wichtig: Wenn man das Kohlblatt auf einem Holzbrett mit einer Holzrolle walkt oder zu einem Brei klopft, empfiehlt es sich, das Blatt in eine Plastiktüte oder zwischen eine Frischhaltefolie zu packen, damit die Gerüche nicht am Holz anhängen bzw. dieses den Saft nicht aufsaugt, der die wirksamste Komponente bei der Anwendung darstellt (Abb. 2; Abb. 3).

Das so vorbereitete Blatt kann nun in der Plastiktüte verpackt zwischen zwei Wärmflaschen erwärmt werden, dann den Brei oder das vorbereitete Blatt auf die saubere Haut auflegen, mit einer Binde fixieren oder einem Tuch umhüllen (Abb. 4; Abb. 5).

Auf offenen Wunden sollte das Kohlblatt nicht über den Wundrand hinausragen, sondern nur in das Wundgebiet eingebracht werden, da der Wundrand sonst aufgeweicht und der Sekret-Abfluss verhindert wird.

\section{Dauer}

Ein Wickel bzw. die Kohlauflage kann bis zu $12 \mathrm{~h}$ aufliegen, bei einem Dekubitus am besten über die Nacht. Wichtig ist eine genaue Beobachtung, wie die Auflage auf die Wunde wirkt.

\section{Praxisbeispiel}

Dorothea F., 80 Jahre alt, hat am rechten Unterschenkel eine Rötung und Stauung. Neben einer medikamentösen Therapie die die Diurese unterstützt, sollten, laut ärztlicher Anordnung, beide Beine täglich vom Pflegedient gewickelt werden. Jeden

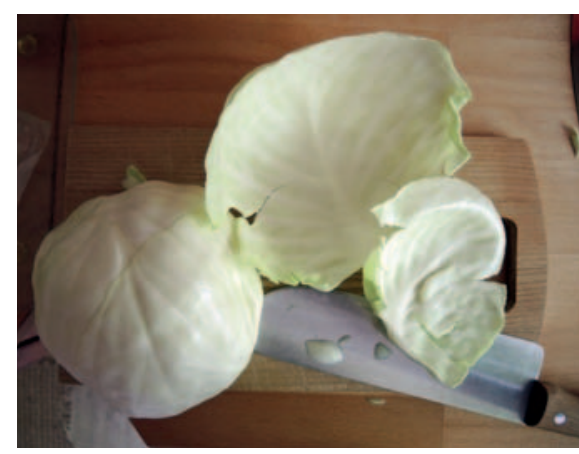

Abb. 1. Nachdem die Weisskohlblätter gewaschen wurden, wird die Mittelrippe ausgeschnitten (Foto: B. Kierey).

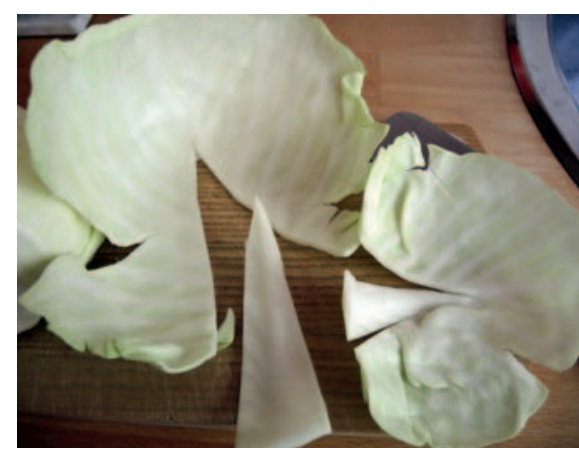

Abb. 2. Die Weisskohlblätter werden zwischen Frischhaltefolie gelegt (Foto: B. Kierey).

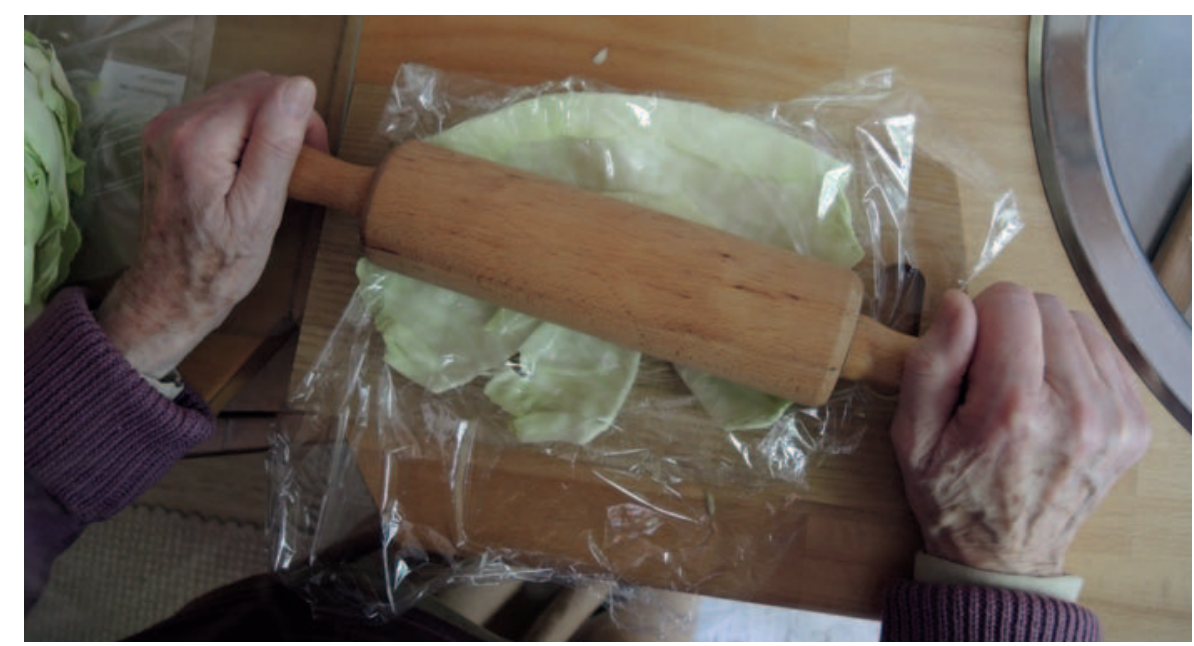

Abb. 3. Mit einem Wellholz werden die benötigten Blätter gewalkt (Foto: B. Kierey). 


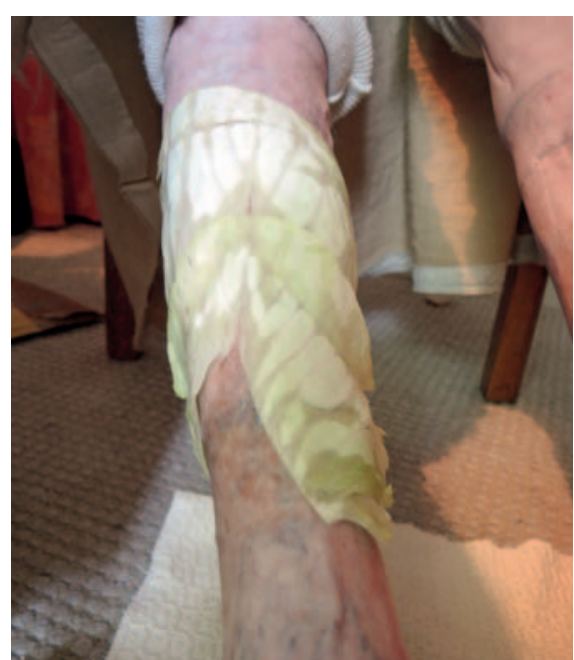

Abb. 4. Die gewalkten Kohlblätter werden auf die Beine aufgelegt (Foto: B. Kierey).

Abend bereitete Frau F. zusätzlich Weisskohlblätter wie oben beschrieben vor, ohne sie jedoch mit zwei Wärmflaschen anzuwärmen. Die vorbereiteten Weisskohlblätter wurden

\section{Literatur}

1 Glaser H: Handbuch der Gesundheitspflege. Alte und neue Hausmittel zur äußeren Anwendung. Esslingen, Gesundheitspflege initiativ, 1999.

2 Brückel I, Deman-Metschies C: WALA Pflege Kompendium, Einführung in die Erwachsenenpflege mit ausgewählten WALA Arzneimitteln, ed 4. Bad Boll/Eckwälden, Wala Heilmittel, 2011.

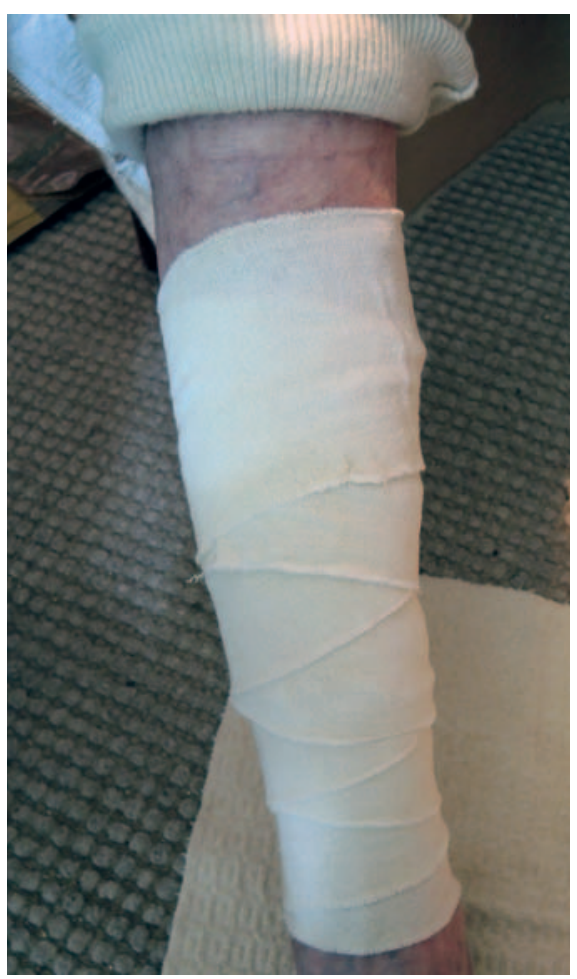

Abb. 5. Anschliessend erfolgt eine Umwicklung der Beine (Foto: B. Kierey).

3 Breit B, Hauser W, Sauer A: Wickel und Auflagen. Gesundheitstipps zum Hausgebrauch. Crailsheim, Deutscher Naturheilverband e.V., 2008

4 Muck H: Rundbrief, Ostern 1994, Verband anthroposophisch orientierter Pflegeberufe e.V., Herdecke. dann vom Pflegepersonal aufgelegt und fixiert. Frau F. nahm die Auflage nach ca. einer Stunde selbständig ab. Insgesamt drei Wochen wurde die Krautauflage durchgeführt. Sie selbst beschreibt die Auflage als «erst kühl, dann sehr angenehm warm» und dass sie ein «anregendes, entlastendes Hautgefühl» erzeuge. Die Unterschenkel zeigten nach dreiwöchiger medikamentöser Therapie und der begleitenden Kohlauflage eine deutliche Entstauung und eine rückläufige Rötung. Anschliessend wurden durch ein Sanitätshaus Kompressionsstrümpfe angepasst und die Beine nun weiter mit Weleda Weiße Malve Pflegelotion rhythmisch eingerieben.

Mithilfe der Weisskohlauflagen als begleitender Pflegemassnahme konnte die abgelagerte, ödematöse Flüssigkeit wieder in den Organismus eingegliedert, in Bewegung und in Fluss gebracht werden.

5 Glaser H: Erfolgreiche Wundbehandlung. Aus der Praxis der anthroposophisch erweiterten Krankenpflege. Stuttgart, Urachhaus, 2000.

6 Fingado M: Therapeutische Wickel und Kompressen. Handbuch aus der Ita Wegman Klinik, ed 2. Dornach, Verlag am Goetheanum, 2003. 